\title{
CONTINUITY AND LINEARITY OF CENTRALIZERS ON A COMPLEMENTED ALGEBRA ${ }^{1}$
}

\author{
PARFENY P. SAWOROTNOW AND GEORGE R. GIELLIS
}

AbSTRACr. Let $A$ be a semisimple complemented algebra and let $T$ be a mapping of $A$ into itself such that either $T(x y)=x T y$ or $T(x y)=(T x) y$ holds for all $x, y \in A$. If $T$ is defined everywhere on $A$ then $T$ is a bounded linear operator.

1. A right centralizer on an algebra $A$ is a mapping $T$ of $A$ into $A$ such that $T(x y)=x T y$ for all $x, y \in A$. A left centralizer is a mapping $T: A \rightarrow A$ such that $T(x y)=(T x) y$ for all $x, y \in A$. This terminology is somewhat different from the terminology of [5] and is due to B. Johnson, who developed the theory of centralizers in [3] and was able to show that for a certain class of Banach algebras centralizers are always linear and bounded. In [4] he showed that this is the case when the algebra has a certain type of bounded approximate identity.

The purpose of this paper is to extend these results of Johnson to the case of complemented algebras. Results of [4] are not applicable to our case. In fact the authors are convinced that Johnson's condition on existence of a certain type of approximate identity in the case of complemented algebras would be equivalent to the assumption of finite-dimensionality of the algebra.

Inasmuch as every proper right $H^{*}$-algebra [8] is a complemented algebra, we have extension of Johnson's result to all types of $H^{*}$-algebras, and in particular, to the algebra of Hilbert Schmidt operators.

We developed our theory for right centralizers but it is obvious that the same theory could be developed for left centralizers.

2. In this section we recall some basic definitions and facts from the theory of complemented algebras. For a more complete background the reader is referred to [6] and [7].

Presented to the Society, October 31, 1970; received by the editors August 19, 1970 and, in revised form, January 10, 1971.

AMS 1970 subject classifications. Primary 46K15; Secondary 47B10.

Key words and phrases. Centralizer, right centralizer, left centralizer, complemented algebra, $H^{*}$-algebra, right $H^{*}$-algebra, left $H^{*}$-algebra, left projection, primitive left projection.

${ }^{1}$ This work was supported by National Science Foundation grant GP-11118. 
A complemented algebra is a Banach algebra $A$ with a Hilbert space norm such that the orthogonal complement of a right (left) ideal is again a right (left) ideal. We say that $x^{l} \in A$ is a left adjoint of $x \in A$ if $(x y, z)=$ $\left(y, x^{l} z\right)$ holds for all $y, z \in A$. A left projection is a nonzero member $e$ of $A$ such that $e^{2}=e^{l}=e$. Note that the inequality $\|e x\| \leqq\|x\|$ holds for each $x \in A$ and any left projection $e$ in $A$ (in this case $(e x, x-e x)=0$ and so $\left.\|x\|^{2}=\|e x\|^{2}+\|x-e x\|^{2}\right)$. Projections $e_{1}, e_{2}$ are said to be doubly orthogonal if $e_{1} e_{2}=e_{2} e_{1}=0$. A primitive left projection is a left projection which cannot be written as a sum of two doubly orthogonal left projections. Each left projection can be written as a sum of a finite number of mutually doubly orthogonal primitive left projections. The right quasiinverse of $a \in A$ is a member $b$ of $A$ such that $a+b+a b=0$ [2]. If a Banach algebra is semisimple then each nonzero ideal contains an element which has no right quasi-inverse [2]. A semisimple complemented algebra $A$ has the property that $A x=0$ implies $x=0$, i.e. in the terminology of Johnson a semisimple complemented algebra is faithful. If $e$ is a primitive left projection and $A_{0}$ is the smallest closed two-sided ideal containing $e$ then $A_{0}$ is a simple algebra.

3. We will need a few lemmas from the theory of complemented algebras. Let $A$ be a fixed semisimple complemented algebra.

LeMma 1. Let $e_{1}, e_{2}$ be primitive left projections belonging to the same minimal closed two-sided ideal $A_{0}$ in $A$. Then the subspaces $e_{i} A e_{j}$ are onedimensional and the subspaces $e_{1} A e_{1}, e_{2} A e_{2}$ are isomorphic to the complex field. Also there are elements $e_{i j} \in a_{i} A e_{j}$ such that $e_{i i}=e_{i}, e_{i j}^{l}=e_{j i}$ and $e_{i j} e_{j k}=e_{i k}$ for all $i, j, k$ in the set $\{1,2\}$.

Proof. First part of the lemma follows from [6, Lemma 7] and the fact that $e_{i} A e_{j}=e_{i} A_{0} e_{j}$. The matrix units $e_{i j}$ can be selected as follows: first set $e_{11}=e_{1}, e_{22}=e_{2}$ and take $e_{12} \in e_{1} A e_{2}$ so that $\left\|e_{12}\right\|=\left\|e_{2}\right\|$; then set $e_{21}=e_{12}^{l}$. The equalities $e_{i j} e_{j k}=e_{i k}$ are then easily verified (compare with pp. 381, 382 of [1]).

\section{LemMa 2. Each nonzero left ideal $L$ in A contains a left projection.}

Proof. We use the technique of [6]. Let $a$ be a member of $L$ which has no right quasi-inverse. Then $-a$ is a relative identity of the proper right ideal $R=$ closure of $\{a x+x \mid x \in A\}$. Write $-a=e+u$ with $e \in R^{p}$, $u \in R$. Then [6, Lemma 2], $e$ is a left projection and $e u=0$. It follows that $e=e(e+u)=e(-a)$ is a member of $L$.

Corollary. Every left ideal in A contains a primitive left projection.

LEMma 3. There exists a family $\left\{e_{\alpha}\right\}_{\alpha \in \Gamma}$ of primitive left projections such that $A=\sum_{\alpha \in \Gamma} A e_{\alpha}$ and $\left(A e_{\alpha}, A e_{\beta}\right)=0$ if $\alpha \neq \beta$ for some $\alpha, \beta \in \Gamma$ (members of $\left\{e_{\alpha}\right\}_{\alpha \in \Gamma}$ do not have to be doubly orthogonal). 
Proof. It is sufficient to consider a maximal family $\left\{e_{\alpha}\right\}$ of primitive left projections such that $\left(A e_{\alpha}, A e_{\beta}\right)=0$ if $\alpha \neq \beta$.

Corollary. If $x \in A$ is such that ex $=0$ for each primitive left projection $e$ in $A$ then $x=0$.

LEMMA 4. Let $e_{1}$ be a primitive left projection in $A$ and let $a \in e_{1} A$. Then there exists a left projection $f \in A$ such that af $=a$.

Proof. Let $A_{0}$ be the smallest closed two-sided ideal containing $e$ and let $f$ be a left projection in the left ideal $A a=A e_{1} a=A_{0} e_{1} a$. Then $f=x e_{1} a$ for some $x \in A$ and there is a primitive left projection $e_{2}$ in $A_{0}$ such that $e_{2} f \neq 0$. Take $e_{12}$ as in Lemma 1 above. Then $e_{12} f=e_{12} x e_{1} a=\lambda e_{1} a$ for some nonzero complex number $\lambda$ (since $e_{1} A e_{1}$ is isomorphic to the complex field). Then $a=e_{1} a=\lambda^{-1} e_{12} f$ and so $a f=a$.

LEMMA 5. If $a, b \in e A$ for some primitive left projection e then there exists $a$ left projection $f$ such that both $a f=a$ and $b f=b$.

Proof. Let $f_{1}, f_{2}$ be left projections such that $a f_{1}=a, b f_{2}=b$. Let $e_{1}, \cdots, e_{n}, e_{n+1}, \cdots, e_{m}$ be primitive left projections such that $f_{1}=\sum_{i=1}^{n} e_{i}$, $f_{2}=\sum_{i=n+1}^{m} e_{i}$. Then the subspaces $e_{i} A e_{j}$ are one dimensional and so the algebra $A^{\prime}$ generated by elements of the form $e_{i} x e_{j}, x \in A$, is a finitedimensional left $H^{*}$-algebra (one can define a left $H^{*}$-algebra in the same way that a right $H^{*}$-algebra was defined in [8]). It is then easy to see that $A^{\prime}$ has an identity $f$ and $f^{l}=f$. It follows then that $a f=a f_{1} f=a f_{1}=a$ and $b f=b f_{2} f=b f_{2}=b$.

4. Now let $T$ be a right centralizer defined everywhere on $A$. Then $T$ is homogeneous [3, Lemma 1, p. 307], i.e. $T(\lambda x)=\lambda T x$ for all $x \in A$ and each complex number $\lambda$. Our aim now is to show that $T$ is a bounded linear operator. This will be done through several lemmas.

Lemma 6. $T$ is linear on $e A$ for any primitive left projection $e$ in $A$.

Proof. We need only to establish the additivity. Let $x, y \in e A$ and let $f$ be a left projection such that $x f=x, y f=y$. Then $T(x+y)=$ $T((x+y) f)=(x+y) T f=x T f+y T f=T(x f)+T(y f)=T x+T y$.

Lemma 7. If a complemented algebra $A$ has a left identity $i$ then $A$ is finite dimensional. The same is true if we assume that $A$ has a right identity.

Proof. First note that the left identity $i$ is a left projection and so $i=\sum_{i=1}^{n} e_{i}$ for some mutually doubly orthogonal primitive left projections $e_{1}, \cdots, e_{n}\left[6\right.$, p. 50]. Then $A=\sum_{i=1}^{n} e_{i} A$ and so it follows from Theorem 1 of [7] that $A$ is a left $H^{*}$-algebra. But then $A=\sum_{i j} e_{i} A e_{j}$ is at most $n^{2}$-dimensional. 
LEMMA 8. Each infinite-dimensional semisimple complemented algebra A contains an infinite family of mutually doubly orthogonal (primitive) left projections.

Proof. Let $\Lambda$ be a maximal family of mutually doubly orthogonal left projections. If $\Lambda$ is finite, say $\Lambda=\left\{e_{1}, e_{2}, \cdots, e_{n}\right\}$, then $e=\sum_{i=1}^{n} e_{i}$ is also a left projection. It follows from Lemma 7 that there exists $a \in A$ such that $a e \neq a$. Then $b=a e-a$ belongs to the left annihilator $l(R)$ of the right ideal $R=\sum_{i=1}^{n} e_{i} A$ and so $l(R) \neq(0)$. Then as in the proof of Lemma 5 of [6] one can show that $R^{p}$ contains a (nonzero) left projection. This would lead to a contradiction with the maximality of $\Lambda$.

Again let $A$ be a semisimple complemented algebra and let $T$ be as above.

LEMMA 9. The restriction of $T$ to $e A$ is a bounded operator, where $e$ is any primitive left projection in $A$.

Proof. Assume that $T$ is not bounded on the unit sphere of $e A$. Then there exists a sequence $\left\{x_{n}\right\}$ of members of $e A$ such that $\left\|x_{n}\right\| \leqq 1$ and $\left\|T x_{n}\right\| \geqq n^{2}$ for all $n$. For each $n$ let $y_{n}=n^{-1} x_{n}$. Then $\left\|y_{n}\right\| \leqq 1 / n$ and $\left\|T y_{n}\right\| \rightarrow \infty$.

Now let $A_{0}$ be the smallest closed two-sided ideal containing $e$. Then $e A=e A_{0}$ is infinite dimensional (otherwise $T$ would be bounded on $e A$ ) and so there exists a sequence $\left\{e_{i}\right\}$ of mutually doubly orthogonal primitive left projections such that $e_{1}=e$. Also one can select as in [6, Theorem 3] a set $\left\{e_{i j}\right\}$ of members of $e_{i} A_{0} e_{j}$ such that $e_{i i}=e_{i}, e_{i j}^{l}=e_{j i}$ and $e_{i j} e_{j k}=e_{i k}$ for all $i, j, k$. Then the series $\sum_{i=1}^{\infty} e_{i 1} y_{i}$ converges to some member $y$ of $A_{0}$ and we have $e_{1 n} y=y_{n}$ for each $n$. It follows then that $\left\|T y_{n}\right\|^{2}=\left\|e_{1 n} T y\right\|^{2}=\left(e_{1 n} T y, e_{1 n} T y\right)=\left(T y, e_{n} T y\right)=\left\|e_{n} T y\right\|^{2} \leqq\|T y\|$ which contradicts the fact that $\left\|T y_{n}\right\| \rightarrow \infty$.

Lemma 10. $T$ is additive on $A$.

Proof. If $x, y \in A$ and $e$ is any minimal left projection then $e T(x+y)=$ $T(e x+e y)=T(e x)+T(e y)=e(T x+T y)$ (because of Lemma 6 above). Thus $T(x+y)=T x+T y$.

Corollary. $T$ is linear on $A$.

Lemma $11 . T$ is bounded on $A$.

Proof. It is sufficient to show that the graph of $T$ is closed. So let $\left\{x_{n}\right\} \subset A$ and $x, y \in A$ be such that $x_{n} \rightarrow x$ and $T x_{n} \rightarrow u$. If $e$ is any primitive left projection then $e x_{n} \rightarrow e x$ and $T\left(e x_{n}\right)=e T x_{n} \rightarrow e u$ and we can also conclude that $T\left(e x_{n}\right)$ tends to $T(e x)=e T x$ (since $T$ is bounded on $e A$ ). Thus $e u=e T x$ for each primitive left projection $e$ and this implies 
that $u=T x$. It follows from the Closed Graph Theorem that $T$ is bounded.

Summing up and taking into account the fact that the same theory can be developed for left centralizers we state our main result.

THEOREM. A right centralizer defined everywhere on a semisimple complemented algebra $A$ is a bounded linear operator on $A$. The same is true for a left centralizer defined everywhere on $A$.

ADDED IN PROOF. It was pointed out to the authors that B. E. Johnson and A. M. Sinclair established continuity of an additive centralizer defined on a semisimple Banach algebra [Amer. J. Math. 90 (1968), 1068-1073]. In the present paper the authors do not assume additivity.

\section{REFERENCES}

1. W. Ambrose. Structure theorems for a special class of Banach algebras, Trans. Amer. Math. Soc. 57 (1945), 364-386. MR 7, 126.

2. N. Jacobson. The radical and semi-simplicity for arbitrary rings, Amer. J. Math. 67 (1945), 300-320. MR 7, 2.

3. B. E. Johnson, An introduction to the theory of centralizers, Proc. London Math. Soc. (3) 14 (1964), 299-320. MR 28 \#2450.

4. - Continuity of centralizers on Banach algebras, J. London Math. Soc. 41 (1966), 639-640. MR 34 \#629.

5. C. N. Kellogg, Centralizers and $H^{*}$-algebras, Pacific J. Math. 17 (1966), 121-129. MR 33 \#1749.

6. P. P. Saworotnow, On a generalization of the notion of $H^{*}$-algebra, Proc. Amer. Math. Soc. 8 (1957), 49-55. MR 19, 47.

7. - On the imbedding of a right complemented algebra into Ambrose's $H^{*}$ algebra, Proc. Amer. Math. Soc. 8 (1957), 56-62. MR 19, 47. 660.

8. M. F. Smiley, Right $H^{*}$-algebras, Proc. Amer. Math. Soc. 4 (1953), 1-4. MR 14,

Department of Mathematics, Catholic University of America, Washington, D.C. 20017

Department of Mathematics, United States Naval Academy, Annapolis, MARYLAND 21402 